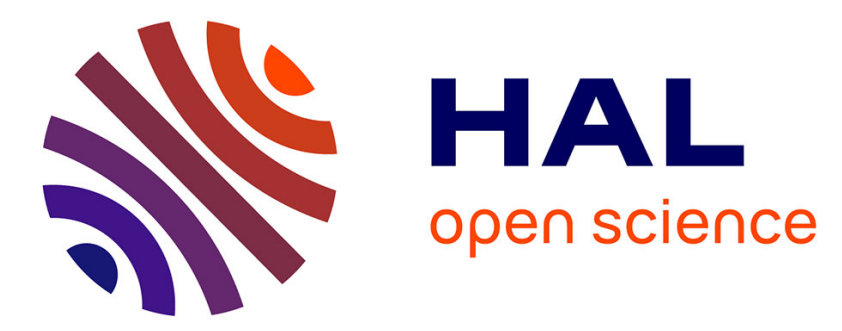

\title{
Structure, magnetic and electrical properties of CeIrSb and its hydride CeIrSbH0.8
}

Etienne Gaudin, Bernard Chevalier, Wilfried Hermes, Ute Ch. Rodewald, Rainer Pöttgen

\section{- To cite this version:}

Etienne Gaudin, Bernard Chevalier, Wilfried Hermes, Ute Ch. Rodewald, Rainer Pöttgen. Structure, magnetic and electrical properties of CeIrSb and its hydride CeIrSbH0.8. Journal of Solid State Chemistry, 2009, 182 (7), pp.1827-1832. 10.1016/j.jssc.2009.04.031 . hal-00406973

\section{HAL Id: hal-00406973 https://hal.science/hal-00406973}

Submitted on 23 Jul 2009

HAL is a multi-disciplinary open access archive for the deposit and dissemination of scientific research documents, whether they are published or not. The documents may come from teaching and research institutions in France or abroad, or from public or private research centers.
L'archive ouverte pluridisciplinaire HAL, est destinée au dépôt et à la diffusion de documents scientifiques de niveau recherche, publiés ou non, émanant des établissements d'enseignement et de recherche français ou étrangers, des laboratoires publics ou privés. 
Structure, magnetic and electrical properties of $\mathrm{CeIrSb}$ and its hydride $\mathrm{CeIrSbH}_{0.8}$

Etienne Gaudin ${ }^{\mathrm{a}}$, Bernard Chevalier ${ }^{\mathrm{a}}$, Wilfried Hermes ${ }^{\mathrm{b}}$, Ute Ch. Rodewald ${ }^{\mathrm{b}}$, and Rainer Pöttgen ${ }^{\mathrm{b}, *}$

a CNRS, Université de Bordeaux, ICMCB, 87 avenue du Docteur Albert Schweitzer, 33608 Pessac Cedex, France

${ }^{b}$ Institut für Anorganische und Analytische Chemie, Westfälische Wilhelms-Universität Münster, Corrensstrasse 30, 48149 Münster, Germany

*Corresponding author. Fax: +49 2518336002 .

E-mail address: pottgen@uni-muenster.de (R. Pöttgen).

\section{A R T I C LE IN F O}

Article history:

Received

Accepted

Available online \#\#\#

Keywords:

Intermetallic compounds

Crystal chemistry

$\mathrm{CeIrSb}$

Kondo system

Metal hydrides

\section{A B S T R A C T}

The ternary antimonide CeIrSb absorbs hydrogen under moderate temperature and pressure conditions ( $4 \mathrm{MPa}$ and $573 \mathrm{~K}$ ), leading to the hydride $\mathrm{CeIrSbH}_{0.8}$. The crystal structures of both compounds have been investigated by X-ray diffraction on powders and single crystals: TiNiSi type, space group Pnma, $a=735.07(7), b=456.93(4), c=$ 
792.8(1) pm, R1/wR2 =0.0206/0.0395, $601 \mathrm{~F}^{2}$ values for CeIrSb and $a=728.16(14), b$ $=460.35(6), c=825.87(2) \mathrm{pm}, \mathrm{R} 1 / w \mathrm{R} 2=0.0322 / 0.0735,528 \mathrm{~F}^{2}$ values for $\mathrm{CeIrSbH}_{0.8}$ with 20 variables per refinement. Hydrogenation induces both an increase of the cell volume $V(+4 \%)$ and a strongly anisotropic expansion of the unit cell with a maximum of $4.3 \%$ in the $c$ direction, leading to a significant increase of the $\mathrm{Ce}-\mathrm{Ir}$ and $\mathrm{Ce}-\mathrm{Ce}$ distances in this direction. The $\mathrm{H}$-insertion into $\mathrm{CeIrSb}$ leads to a magnetic transition from intermediate valence to antiferromagnetic behavior $\left(\mathrm{T}_{\mathrm{N}}=7.0 \mathrm{~K}\right)$ evidenced by magnetization, electrical resistivity and specific heat measurements. This transition can be explained on the basis of the Doniach diagram considering the $J_{c f}$ interaction between the $4 f(\mathrm{Ce})$ and conduction electrons.

(c) 2009 Elsevier Ltd. All rights reserved.

\section{Introduction}

The equiatomic cerium-transition metal $(T)$-antimonides CeTSb exist with $T=\mathrm{Ni}$, $\mathrm{Rh}, \mathrm{Pd}, \mathrm{Ir}, \mathrm{Pt}$ and $\mathrm{Au}$ [1]. They crystallize with different superstructures of the hexagonal $\mathrm{AlB}_{2}$ type. The degree of puckering of the distorted $T_{3} \mathrm{Sb}_{3}$ hexagons depends on the valence electron concentration (VEC). CeTSb with $T=\mathrm{Ni}$ [2], $\mathrm{Pd}$ [3], $\mathrm{Pt}$ [4], and $\mathrm{Au}[5,6]$ adopt hexagonal structures (ZrBeSi or NdPtSb type) with planar or slightly puckered $T_{3} \mathrm{Sb}_{3}$ hexagons. Detailed single crystal studies on these ternary antimonides, however, have not been performed. CeRhSb [7-9] and CeIrSb [10-12] with the lower VEC adopt the orthorhombic TiNiSi type structure with strongly tilted and puckered $\mathrm{Rh}_{3} \mathrm{Sb}_{3}$ and $\mathrm{Ir}_{3} \mathrm{Sb}_{3}$ hexagons.

The magnetic and electrical properties of the $\mathrm{CeTSb}$ ternary antimonides have intensively been studied in recent years. $\mathrm{CeNiSb}$ and $\mathrm{CePdSb}$ are ferromagnetic Kondolattice systems [13, 14] and CePtSb orders ferromagnetically below 4.5 K [15]. Magnetic ordering of CeAuSb at $4.2 \mathrm{~K}$ was evident from specific heat data [5]. The most interesting compounds are the valence fluctuating systems $\mathrm{CeRhSb}$ [7] and CeIrSb [11]. CeRhSb is a so-called Kondo semiconductor and exhibits opening at low temperatures of a small pseudogap in the electronic density of states. So far more than 85 entries occur for CeRhSb in the current SciFinder Scholar version [16]. On the contrary, it was suggested that CeIrSb is a valence fluctuating compound with stronger 
$4 f(\mathrm{Ce})$-conduction electrons hybridization than that for $\mathrm{CeRhSb}$ [11]. But a recent investigation of CeIrSb by Sb NMR reveals that this compound can be classified as $\mathrm{CeRhSb}$ into the same group revealing a V-shaped gap due to $4 f(\mathrm{Ce})$-conduction electrons hybridization [12]. Finally, it was recently reported that the hydrogenation of $\mathrm{CeRhSb}$ leads to the formation of the new hydride $\mathrm{CeRhSbH}_{0.2}$ which orders antiferromagnetically at $3.6 \mathrm{~K}$ [9]. Electrical resistivity and specific heat measurements point to a considerable Kondo effect in the hydride.

In spite of the extensive studies on $\mathrm{CeRhSb}$, only little information on isotypic CeIrSb is available in the literature [10-12, 17]. Structural information on CeIrSb relies only on powder X-ray data. In continuation of our recent work on $\mathrm{CeRhSb}$, we were interested in the influence of the hydrogenation on the structural and physical properties of CeIrSb. Herein we report on the synthesis and properties of the new hydride $\mathrm{CeIrSbH}_{0.8}$ and a single crystal structure refinement of the parent compound CeIrSb. The antiferromagnetic hydride was also characterized by electrical resistivity, specific heat and magnetization measurements.

\section{Experimental}

\subsection{Synthesis}

The starting materials for the preparation of CeIrSb were cerium ingots (smart elements), iridium powder (Heraeus) and antimony lumps (Johnson-Matthey), all with a stated purity better than $99.9 \%$. The cerium ingots were first cut into smaller pieces and arc-melted [18] into small buttons under an argon atmosphere of approximately 600 mbar. The argon was purified with molecular sieves, silica gel, and titanium sponge $(900 \mathrm{~K})$. The pre-melting procedure of cerium strongly reduces shattering during the exothermic reaction with iridium and antimony. The iridium powder was cold-pressed to a pellet $(\varnothing=6 \mathrm{~mm})$. The elements were then weighed in the ideal 1:1:1 atomic ratio and reacted by arc-melting under argon. The product ingot was remelted three times in order to ensure homogeneity. The total weight loss after the melting procedures was smaller than $0.5 \%$. The resulting CeIrSb sample was polycrystalline.

For the growth of small single crystals, an arc-melted CeIrSb button was sealed in an evacuated silica tube and placed into the center of a water cooled quartz sample chamber of an induction furnace (Hüttinger Elektronik, Freiburg, type TIG 1.5/300) 
equipped with a levitation coil-set. The sample was first carefully heated up to the (nondetermined) softening point (close to the melting temperature). The power output of the generator was then lowered by ca. $10 \%$ and the sample was kept at that temperature for another 4 hours, followed by switching off the power supply.

The polycrystalline and the annealed CeIrSb sample are silvery with metallic luster and stable in air over months.

\subsection{Hydrogenation reaction}

Hydrogen absorption experiments were performed using the apparatus described previously [19]. An annealed ingot of CeIrSb was heated under vacuum at $573 \mathrm{~K}$ for $2 \mathrm{~h}$ and then exposed to $4 \mathrm{MPa}$ of hydrogen gas at the same temperature for two days. The hydrogenation induces a decrepitation of the starting ingot. The amount of hydrogen absorbed was determined volumetrically by monitoring pressure changes in a calibrated volume. Under these conditions, the new hydride $\mathrm{CeIrSbH}_{0.8}$ was obtained. The latter is stable in ambient conditions.

\subsection{EDX analyses}

The phase purity of the bulk samples and of the single crystal of CeIrSb investigated on the diffractometer was analyzed using a Leica 420I scanning electron microscope. $\mathrm{CeO}_{2}$, iridium, and $\mathrm{Sb}$ were used as standards for the semiquantitative EDX analysis. No impurity elements heavier than sodium were observed. The experimentally determined compositions were very close to the ideal one.

\subsection{X-ray image plate data and data collection}

The polycrystalline CeIrSb sample was analyzed by powder X-ray diffraction on a Guinier camera (equipped with an image plate system Fujifilm, BAS-1800) using Cu $\mathrm{K} \alpha_{1}$ radiation and $\alpha$-quartz $(a=491.30, c=540.46 \mathrm{pm})$ as an internal standard. The orthorhombic lattice parameters (Table 1) were obtained from least-squares refinements of the powder data. The correct indexing of the diffraction lines was ensured by an intensity calculation [20] using the positional parameters obtained from the structure refinement. Our data compare well with the ones from Haase et al. ( $a=736.0(1), b=$ 457.8(1) and $c=793.1(2) \mathrm{pm})$ [10] and Sasakawa et al. $(a=735.1, b=457.4$ and $c=$ $793.5 \mathrm{pm})[11]$. 
Irregularly shaped crystal fragments of $\mathrm{CeIrSb}$ were obtained from the larger ingot annealed in the levitation furnace by mechanical fragmentation. The crystals were glued to quartz fibres usings bees wax and were investigated by Laue photographs on a Buerger camera (white molybdenum radiation, image plate technique, Fujifilm, BAS1800) in order to check the quality for intensity data collection. The data set was collected at room temperature by use of an IPDS II diffractometer (graphite monochromatized Mo $K \alpha$ radiation; oscillation mode). A numerical absorption correction was applied to the data set. All relevant crystallographic data and details of the data collection and evaluation are listed in Table 1.

X-ray powder diffraction with the use of a Philips 1050 diffractometer $(\mathrm{Cu} \mathrm{K \alpha}$ radiation) was applied for the characterization of the structural type and for the phase identification of the hydride sample. The unit cell parameters were determined by a least-squares refinement method using silicon $(5 N)$ as an internal standard. The refinement of the crystal structure of $\mathrm{CeIrSbH}_{0.8}$ was performed using a tiny single crystal isolated from the crushed sample and selected by optical microscopy. Reflection data were collected at room temperature on an Enraf-Nonius Kappa charge coupled device (CCD) area-detector diffractometer using Mo $K \alpha$ radiation. A Gaussian-type absorption correction was applied, the shape of the crystal being determined with the video microscope of the diffractometer. Data processing and all refinements were performed with the JANA2006 program package [21]. Details of data collections and structure refinements are listed in Table 1.

\subsection{Physical properties measurements}

For electrical resistivity investigation, the hydride $\mathrm{CeIrSbH}_{0.8}$ was compacted at room temperature (compactness $\approx 80 \%$ ) in order to form a polycrystalline pellet $(\varnothing=6$ $\mathrm{mm}$ and thickness $=3 \mathrm{~mm}$ ) and then heated for 2 days at $573 \mathrm{~K}$ under pressure $(4 \mathrm{MPa})$ of hydrogen. After this thermal treatment, which improves the mechanical behavior, the pellet was checked by X-ray diffraction; no structural change was evidenced. For electrical resistivity measurements, a bar of $1.5 \times 1.5 \times 5 \mathrm{~mm}^{3}$ was cut from the pellet. The measurements were carried out above $4.2 \mathrm{~K}$ using the standard dc four probe method with silver paint contacts and an intensity current of $10 \mathrm{~mA}$. Finally, magnetization measurements were performed on a part of the powered pellet using a 
superconducting quantum interference device magnetometer in the temperature range of $1.8-300 \mathrm{~K}$ and applied fields up to $4.6 \mathrm{~T}$.

Heat capacity measurements on the hydride $\mathrm{CeIrSbH}_{0.8}$ were performed by a relaxation method with a Quantum Design PPMS system and using a two tau model analysis. Data were taken in the $2.2-40 \mathrm{~K}$ temperature range. For these latter measurements, the sample was a plate of $28.2 \mathrm{mg}$ weight obtained from the same pellet used for the other physical characterization.

\section{Results and discussion}

\subsection{Structure refinements}

The diffractometer data set of CeIrSb showed a primitive orthorhombic cell and the systematic extinctions were compatible with space group Pnma, similar to our recent investigation on isotypic TiNiSi type CeRhSb [9]. The atomic parameters of $\mathrm{CeRhSb}$ [9] were taken as starting parameters and the structure was refined using SHELXS-97 [22] (full-matrix least-squares on $\mathrm{F}^{2}$ ) with anisotropic atomic displacement parameters for all atoms. As a check for the correct composition, the occupancy parameters were refined in a separate series of least-squares cycles. All sites were fully occupied within two standard uncertainties and in the final cycles the ideal occupancies were assumed again. The positional parameters and interatomic distances are listed in Tables 2 and 3.

The extinction conditions observed for the $\mathrm{CeIrSbH}_{0.8}$ single-crystal agree with the Pnma space group already used for the refinement of the initial ternary antimonides $\mathrm{CeIrSb}$ or $\mathrm{CeRhSb}$. The starting atomic positions were those reported here for CeIrSb (Table 2). With anisotropic displacement parameters, the final residual factors converged to the values $R(F)=0.032$ and $w R\left(F^{2}\right)=0.074$ for twenty refined parameters and 528 used reflections (Table 1). The final atomic positions and anisotropic displacement parameters are given in Table 2. The diffraction data did not allow for a location of the hydrogen sites.

Further details on the structure refinements are available from Fachinformationszentrum Karlsruhe, D-76344 Eggenstein-Leopoldshafen (Germany), by quoting the Registry No's. CSD-420488 (CeIrSb) and CSD-380302 (CeIrSbH $\left.{ }_{0.8}\right)$. 


\subsection{Crystal chemistry}

$\mathrm{CeIrSb}$ as CeRhSb crystallizes with the orthorhombic TiNiSi type structure [9]. Since the crystal chemistry and chemical bonding of TiNiSi type compounds has intensively been discussed in various review articles [23-27], herein we focus only on the structural peculiarities of CeIrSb with respect to the hydride formation. The cerium near-neighbor coordination of $\mathrm{CeIrSb}$ and $\mathrm{CeIrSbH}_{0.8}$ is presented in Fig. 1. Each Ce atom has six Ir, $\mathrm{six} \mathrm{Sb}$, and four Ce atoms in the first coordination shell. The $\mathrm{Ir}$ and $\mathrm{Sb}$ atoms form ordered $\mathrm{Ir}_{3} \mathrm{Sb}_{3}$ hexagons which build up a sandwich-like arrangement around the $\mathrm{Ce}$ atoms. In CeIrSb, the Ir-Sb distances range from 268 to $283 \mathrm{pm}$ (Table 3), comparable to the sum of the covalent radii of $267 \mathrm{pm}$ [28]. Bonding of the Ce atoms to the threedimensional [IrSb] network proceeds through the Ce-Ir contacts. The closest Ce-Ir distance at $304 \mathrm{pm}$ (Table 3) is only slightly longer than the sum of the covalent radii of 291 pm [28]. Similar short Ce-Ir distances (295 and 305 pm) also occur in isotypic CeIrGe which presents an intermediate valence state [29].

The CeIrSb structure leaves several tetrahedral voids $\left[\mathrm{Ce}_{3} \mathrm{Ir}\right]$ which can be filled with hydrogen. Similar H-insertion was reported for the ternary compounds adopting the TiNiSi type as CeNiSn [30] or NdNiSn [31]. Upon hydrogenation of CeIrSb, we observe an increase of the cell volume $V$ by $4.3 \%$, however, in a strongly anisotropic manner. The $a$ lattice parameter decreases by $0.7 \%$ while the $b$ and $c$ parameters increase by 0.7 and $4.3 \%$, respectively. In line with the anisotropic expansion we observe an increase of the interatomic distances in this direction. All Ce-Ir distances increase by 1 up to $12 \mathrm{pm}$ (Table 3 ), indicating a decrease of the $4 f(\mathrm{Ce})-5 d(\mathrm{Ir})$ hybridization. The most pronounced increase concerns the $\mathrm{Ce}-\mathrm{Ce}$ distances. As is evident from Fig. 1, the Ce-Ce distance in the $c$ direction increases by 21 pm from 384 to $405 \mathrm{pm}$, while only a minor decrease of $4 \mathrm{pm}$ is realized in the $a$ direction. These changes have drastic influence on the physical properties ingoing from intermediate valent CeIrSb to the hydride $\mathrm{CeIrSbH}_{0.8}$ (vide infra).

The metal atoms of the hydride $\mathrm{CeIrSbH}_{0.8}$ adopt almost the same structure as the initial compound CeIrSb. This behavior is comparable to that observed during the hydrogenation of the other orthorhombic ternary compounds as CeNiSn [30, 32-36], CePdSn [37] and CeRhSb [9]. The resulting hydrides exhibit the same structure than 
$\mathrm{CeIrSbH}_{0.8}$. In this structure, two types of chemically similar but geometrically different $\left(\mathrm{Ce}_{3} T\right.$ with $T=\mathrm{Ni}, \mathrm{Pd}, \mathrm{Rh}$ ) tetrahedra are formed; only one that has a nearly regular $\mathrm{Ce}_{3}$ side is occupied by a hydrogen atom [30]. The other significantly deformed $\left(\mathrm{Ce}_{3} T\right)$ tetrahedron is empty.

\subsection{Physical properties}

The temperature dependence of the reduced resistivity $\rho(\mathrm{T}) / \rho(270 \mathrm{~K})$ of the hydride $\mathrm{CeIrSbH}_{0.8}$ reveals several characteristics (Fig. 2): (i) a downward curvature around 150-200 K; such behaviour, which is similar with that reported previously for $\mathrm{CeNiSnH}_{1.0}$ [35], is expected for Kondo-type interactions in the presence of crystal field effects [38]; (ii) a minimum around $30 \mathrm{~K}$ and a maximum near $8 \mathrm{~K}$; the decrease of $\rho(T) / \rho(270 \mathrm{~K})$ below this last temperature could be associated with the loss of spin disorder scattering of the conduction electrons owing to the occurrence of a magnetic transition. No similar decrease is observed from the curve $\rho=\mathrm{f}(\mathrm{T})$ for CeIrSb [11]; this last curve characterizes a valence-fluctuating system.

The temperature dependence of the reciprocal magnetic susceptibility $\chi_{\mathrm{m}}{ }^{-1}$ of CeIrSb (inset of Fig. 3) is in qualitative agreement with the data reported previously [11]. Below $300 \mathrm{~K}$, in any temperature range, the curve $\chi_{\mathrm{m}}{ }^{-1}=\mathrm{f}(\mathrm{T})$ can be explained by a Curie-Weiss law on account of the intermediate valence behaviour of CeIrSb. On the contrary, this curve follows a modified Curie-Weiss law $\chi_{\mathrm{m}}{ }^{-1}=\chi_{0}{ }^{-1}+\left(\mathrm{T}-\theta_{\mathrm{p}}\right) / \mathrm{C}_{\mathrm{m}}$ above 5 $\mathrm{K}$, leading to an effective paramagnetic moment $\mu_{\mathrm{eff}}=\left(8 \mathrm{C}_{\mathrm{m}}\right)^{1 / 2}=0.8 \mu_{\mathrm{B}} / \mathrm{mol}$ and a small positive paramagnetic Curie temperature $\theta_{p}=3 \mathrm{~K}$ (see the red line in the inset of Fig. $3)$. The temperature independent term $\chi_{0}$ is equal to $1.3 \times 10^{-3} \mathrm{emu} / \mathrm{mol}$. The relatively large value of $\chi_{0}$, attributed to the strong $4 f(\mathrm{Ce})$-conduction electrons hybridization, considerably distorts the relation $\chi_{\mathrm{m}}{ }^{-1}=\mathrm{f}(\mathrm{T})$ from a straight line, producing a large negative curvature as $\mathrm{T}$ decreases. We recall that $\chi_{0}$ should include the diamagnetic core contribution, the Pauli susceptibility of the conduction electrons and the Van Vleck temperature independent paramagnetism. The value of $\mu_{\text {eff, which is very low compared }}$ with that calculated for $\mathrm{Ce}^{3+}\left(2.54 \mu_{\mathrm{B}}\right)$, is consistent with a strong $4 f(\mathrm{Ce})$-conduction electrons interaction inducing an intermediate valence behaviour. 
On the contrary, above $100 \mathrm{~K}$, the $\chi_{\mathrm{m}}{ }^{-1}=\mathrm{f}(\mathrm{T})$ data relative to the hydride $\mathrm{CeIrSbH}_{0.8}$ can be fitted with a Curie-Weiss law $\chi_{\mathrm{m}}{ }^{-1}=\left(\mathrm{T}-\theta_{\mathrm{p}}\right) / \mathrm{C}_{\mathrm{m}}$ (Fig. 3) giving $\theta_{\mathrm{p}}=-142 \mathrm{~K}$ and $\mu_{\text {eff }}=2.67 \mu_{\mathrm{B}} / \mathrm{mol}$; a value close to that calculated for $\mathrm{Ce}^{3+}$ ion. The negative curvature observed at low temperatures in the $\chi_{\mathrm{m}}{ }^{-1}=\mathrm{f}(\mathrm{T})$ curve indicates the presence of a crystalfield effect. In other words, hydrogenation of the ternary antimonide CeIrSb induces a change of the valence state of Ce from intermediate to purely trivalent. Similar behavior was observed previously by hydrogenation of the Kondo semiconductor $\mathrm{CeRhSb} \mathrm{[9].}$

At low temperature, the thermal dependence of the magnetization of $\mathrm{CeIrSbH}_{0.8}$, presented in Fig. 4, shows the occurrence of a shoulder near 7.0(2) K. This behaviour suggests that the hydride orders antiferromagnetically at this last temperature. The magnetic ordering is further corroborated by the field-dependent behaviour of the magnetization taken below $\mathrm{T}_{\mathrm{N}}=7.0 \mathrm{~K}$ (Fig. 5). For instance, at $4 \mathrm{~K}$ and up to $\mathrm{H} \leq 4.6 \mathrm{~T}$, in the antiferromagnetic range, the magnetization of $\mathrm{CeIrSbH}_{0.8}$ increases almost linearly at low magnetic fields and more rapidly above $3.5 \mathrm{~T}$. This behaviour reveals the appearance of a magnetic transition induced by the applied magnetic field as observed in many antiferromagnets (spin-flip or spin-flop transition). This metamagnetic transition depends on temperature since it is not observed at $6 \mathrm{~K}$ but appears at $2 \mathrm{~K}$. At this temperature, the curvature evidenced for $0 \leq \mathrm{H} \leq 3.5 \mathrm{~T}$ in the curve $\mathrm{M}=\mathrm{f}(\mathrm{H})$ (Fig. 5 ) is not intrinsic but can be associated to the presence of some amounts of impurity phase $\mathrm{CeSb}$ not detected by X-ray powder analysis. It is well known that $\mathrm{CeSb}$ orders antiferromagnetically below $16 \mathrm{~K}$ and exhibits a complex magnetic phase diagram [39]. Finally, it should be mentioned that the hydrogenation of CeIrSb leads as in the case of $\mathrm{CeRhSb}$ [9] to an antiferromagnet hydride but the Néel temperature of the hydride $\mathrm{CeIrSbH}_{0.8}$ is twice higher than that determined for $\mathrm{CeRhSbH}_{0.2}\left(\mathrm{~T}_{\mathrm{N}}=3.6 \mathrm{~K}\right)$. The comparison of these two hydrides indicates that: (i) CeIrSb absorbs in the same conditions more hydrogen and (ii) the resulting increase of the cell volume $V$ is greater; during the hydrogenation of $\mathrm{CeIrSb}$ and $\mathrm{CeRhSb}$ this volume increase is respectively equal to 4.3 and $0.5 \%$.

As indicated in ref. [9], the hydrogenation of these equiatomic antimonides leads to an expansion of $V$ which favours a decrease of the hybridization $J_{c f}$ between the $4 f(\mathrm{Ce})$ and the conduction electrons. The magnetic Ruderman-Kittel-Kasuya-Yosida (RKKY) 
interaction, which drives the compound towards a long-range magnetic ordering, increases whereas the Kondo interaction, which tends to demagnetize the $4 f(\mathrm{Ce})$ states, decreases $[32,37]$. In other words, in these cases the hydrogenation which transforms an intermediate valence compound ( $\mathrm{CeIrSb})$ or a Kondo semiconductor ( $\mathrm{CeRhSb})$ into an antiferromagnet $\left(\mathrm{CeIrSbH}_{0.8}\right.$ or $\left.\mathrm{CeRhSbH}_{0.2}\right)$ can be compared to the application of a "negative" pressure inducing a decrease of the $J_{c f}$ interaction. This effect can be explained on the basis of the Doniach diagram [37]. Also a higher increase of the cell volume $V$ induces a greater Néel temperature as observed in the sequence $\mathrm{CeRhSbH}_{0.2}$ $\left(\mathrm{T}_{\mathrm{N}}=3.6 \mathrm{~K}\right) \rightarrow \mathrm{CeIrSbH}_{0.8}\left(\mathrm{~T}_{\mathrm{N}}=7.0 \mathrm{~K}\right)$.

Specific heat $\mathrm{C}_{\mathrm{p}}$ measurements provide further support to the occurrence of an antiferromagnetic ordering for $\mathrm{CeIrSbH}_{0.8}$. As shown in Fig. 6, there exists a clear $\lambda$ type anomaly at about $6.9(2) \mathrm{K}$ in the $\mathrm{C}_{\mathrm{p}}$ vs $\mathrm{T}$ curve; the peak value approaching 2.32 $\mathrm{J} / \mathrm{K}$ mol. The Néel temperature determined by this investigation agrees with that evidenced previously by magnetization measurements $\mathrm{T}_{\mathrm{N}}=7.0(2) \mathrm{K}$. Valuable information can be obtained from the magnetic entropy associated with the antiferromagnetic ordering of $\mathrm{CeIrSbH}_{0.8}$, which is estimated from the magnetic contribution to $C_{p}$ in the low temperature range $(T \leq 22.5 \mathrm{~K})$ as $C_{p \text { mag }}=C_{p}-\left(\gamma+\beta T^{2}\right)$. The electronic coefficient $\gamma$ and phonon constant $\beta$ were estimated by fitting of the $C_{p} / T$ vs $\mathrm{T}^{2}$ plot for $11 \leq \mathrm{T} \leq 22.5 \mathrm{~K}$ (inset of Fig. 6). This procedure gives $\gamma=63 \mathrm{~mJ} / \mathrm{K}^{2} \mathrm{~mol}$ and $\beta=0.522 \mathrm{~mJ} / \mathrm{K}^{4}$ mol. At $\mathrm{T}_{\mathrm{N}}$, the magnetic entropy reaches $1.90 \mathrm{~J} / \mathrm{K} \mathrm{mol}=0.33$ $\mathrm{R} \ln 2$, which is substantially reduced from $\mathrm{R} \ln 2=5.76 \mathrm{~J} / \mathrm{K}$ mol, the value of magnetic entropy expected for a doublet ground state of $\mathrm{Ce}^{3+}$. This reduction which is lower than that reported for $\mathrm{CeRhSbH}_{0.2}(0.22 \mathrm{R} \ln 2)$ [9], suggests the presence of a Kondo effect in the hydride $\mathrm{CeIrSbH}_{0.8}$. This agrees with the significant value of the electronic term $\gamma=$ $63 \mathrm{~mJ} / \mathrm{K}^{2}$ mol. It should be mentioned that this $\gamma$-value is higher than that determined for the initial intermediate valence compound CeIrSb $\left(\gamma=10 \mathrm{~mJ} / \mathrm{K}^{2} \mathrm{~mol}\right)$ [11]. In this case, the small $\gamma$-value implies that the $4 f(\mathrm{Ce})$ electronic states are strongly hybridized with the conduction band. The increase of the $\gamma$-value in the sequence $\mathrm{CeIrSb} \rightarrow$ $\mathrm{CeIrSbH}_{0.8}$ agrees with a decrease of the $J_{c f}$ interaction leading to a transition from a non magnetic state to a magnetic state as observed in the strongly correlated electron systems $[40,41]$. 


\section{Conclusion}

As reported previously for $\mathrm{CeNiSn}, \mathrm{CePdSn}$ and $\mathrm{CeRhSb}$, the hydrogenation of $\mathrm{CeIrSb}$ produces a hydride adopting the same structure to the initial equiatomic ternary compound. This hydrogenation which induces an expansion the cell volume provokes the transition from intermediate valence (CeIrSb) to antiferromagnetic behaviour $\left(\mathrm{CeIrSbH}_{0.8}\right)$. But the investigation of this hydride by electrical resistivity and specific heat measurements reveals that the Kondo effect plays a role on its physical properties. $\mathrm{CeIrSbH}_{0.8}$ exhibits the higher Néel temperature $\mathrm{T}_{\mathrm{N}}=7.0 \mathrm{~K}$ among the hydrides crystallizing in the orthorhombic TiNiSi type as $\mathrm{CeRhSbH}_{0.2}\left(\mathrm{~T}_{\mathrm{N}}=3.6 \mathrm{~K}\right)$ [9], $\mathrm{CeNiSnH}$ $\left(\mathrm{T}_{\mathrm{N}}=4.5 \mathrm{~K}\right)$ [32] and CePdSnH $\left(\mathrm{T}_{\mathrm{N}}=5.0 \mathrm{~K}\right)$ [37]. The high value of $\mathrm{T}_{\mathrm{N}}$ for $\mathrm{CeIrSbH}_{0.8}$ can be correlated to the increase of the cell volume $V$ observed during the hydrogenation of CeIrSb; in going from CeIrSb to $\mathrm{CeIrSbH}_{0.8}$, the increase of $V$ is about $4.3 \%$ and is greater than those detected during the $\mathrm{H}$-insertion into CeRhSb, CePdSn or CeNiSn respectively equal to $0.5,2.5$ and $2.6 \%[9,32,37]$. This present study confirms that the hydrogenation of the intermetallics based on the cerium modifies strongly the competition between the RKKY magnetic interaction and the demagnetizing Kondo interaction which governs their physical properties.

\section{Acknowledgements}

This work was financially supported by the Deutsche Forschungsgemeinschaft. B.C., E.G. and R.P. are indebted to EGIDE and DAAD for research grants within the PROCOPE programs (11457RD and D/0502176). Finally, B.C. thanks the European Science Foundation (ECOM-COST action P16) for financial support. W.H. is indebted to the Fonds der Chemischen Industrie and the NRW Graduate School of Chemistry for a $\mathrm{PhD}$ stipend.

\section{References}

[1] O.L. Sologub, P.S. Salamakha, Rare earth-antimony systems, in: K.A. Gschneidner Jr., J.-C.G. Bünzli, V.K. Pecharsky (Eds.), Handbook on the Physics and Chemistry of Rare Earths, vol. 33, Elsevier, Amsterdam, 2003, pp. 35-146 (Chapter 212). 
[2] K. Hartjes, W. Jeitschko, J. Alloys Compd. 226 (1995) 81-86.

[3] R. Marazza, D. Rossi, R. Ferro, J. Less-Common Met. 75 (1980) P25-P28.

[4] D. Rossi, R. Marazza, D. Mazzone, R. Ferro, J. Less-Common Met. 78 (1981) P1P5.

[5] H. Suzuki, T. Yamaguchi, K. Katoh, M. Kasaya, Physica B 186-188 (1993) 390392.

[6] L.P. Salamakha, O.L. Sologub, A.P. Gonçalves, S.I. Mudryi, V.B. Kapustianyk, M. Almeida, J. Alloys Compd. 430 (2007) 175-178.

[7] S.K. Malik, D.T. Adroja, Phys. Rev. B 43 (1991) 6277-6279.

[8] P. Salamakha, O. Sologub, T. Suemitsu, T. Takabatake, J. Alloys Compd. 313 (2000) L5-L9.

[9] B. Chevalier, R. Decourt, B. Heying, F. M. Schappacher, U. Ch. Rodewald, R.-D. Hoffmann, R. Pöttgen, R. Eger, A. Simon, Chem. Mater. 19 (2007) 28-35.

[10] M.G. Haase, T. Schmidt, C.G. Richter, H. Block, W. Jeitschko, J. Solid State Chem. 168 (2002) 18-27.

[11] T. Sasakawa, K. Shigetoh, D. Hirata, K. Umeo, T. Takabatake, Physica B 359-361 (2005) 111-114.

[12] Y. Kawasaki, M. Izumi, Y. Kishimoto, T. Ohno, H. Tou, Y. Inaoka, M. Sera, K. Shigetoh, T. Takabatake, Phys. Rev. B 75 (2007) 094410-5.

[13] L. Menon, S. K. Malik, Phys. Rev. B 52 (1995) 35-38.

[14] S. K. Malik, D. T. Adroja, Phys. Rev. B 43 (1991) 6295-6298.

[15] B. D. Rainford, D. T. Adroja, Physica B 194-196 (1994) 365-366.

[16] 88 entries occur for the formula CeRhSb in the SciFinder Scholar version 2008: http://www.cas.org/SCIFINDER/SCHOLAR/

[17] Y. Kawasaki, T. Tanaka, M. Izumi, Y. Kishimoto, T. Ohno, H. Tou, Y. Inaoka, M. Sera, K. Shigetoh, T. Takabatake, J. Phys. Chem. Solids 68 (2007) 2195-2198.

[18] R. Pöttgen, Th. Gulden, A. Simon, GIT Labor-Fachzeitschrift 43 (1999) 133-136.

[19] J.-L. Bobet, S. Pechev, B. Chevalier, B. Darriet, J. Alloys Compd. 267 (1998) 136141.

[20] K. Yvon, W. Jeitschko, E. Parthé, J. Appl. Crystallogr. 10 (1977) 73-74.

[21] V. Petricek, M. Dusek and L. Palatinus, Jana 2006 The crystallographic computing system, Institute of Physics, Praha, Czech Republic, 2006.

[22] G. M. Sheldrick, SHELXS-97 - Program for the Solution of Crystal Structures, University of Göttingen, Germany, 1997.

[23] G. Nuspl, K. Polborn, J. Evers, G.A. Landrum, R. Hoffmann, Inorg. Chem. 35 (1996) 6922-6932.

[24] G.A. Landrum, R. Hoffmann, J. Evers, H. Boysen, Inorg. Chem. 37 (1998) 57545763. 
[25] R.-D. Hoffmann, R. Pöttgen, Z. Kristallogr. 216 (2001) 127-145.

[26] M.D. Bojin, R. Hoffmann, Helv. Chim. Acta 86 (2003) 1653-1682.

[27] E. Parthé, L. Gelato, B. Chabot, M. Penzo, K. Cenzual, R. Gladyshevskii, TYPIX-Standardized Data and Crystal Chemical Characterization of Inorganic Structure Types. Gmelin Handbook of Inorganic and Organometallic Chemistry, 8th edition, Springer, Berlin (Germany), 1993.

[28] J. Emsley, The Elements, Oxford University Press, Oxford (U.K.), 1999.

[29] E. Gaudin, B. Chevalier, B. Heying, U.Ch. Rodewald, R. Pöttgen, Chem. Mater. 17 (2005) 2693-2700.

[30] V. A. Yartys, B. Ouladdiaf, O. Isnard, O. Khyzhun, K. H. J. Buschow, J. Alloys Compd. 359 (2003) 62-65.

[31] V. A. Yartys, T. Olavesen, B. C. Hauback, H. Fjellvag, J. Alloys Compd. 336 (2002) 181-186.

[32] B. Chevalier, M. Pasturel, J.-L. Bobet, R. Decourt, J. Etourneau, O. Isnard, J. Sanchez Marcos, J. Rodriguez Fernandez, J. Alloys Compd. 383 (2004) 4-9.

[33] B. Chevalier, J.-L. Bobet, M. Pasturel, E. Bauer, F. Weill, R. Decourt, J. Etourneau, Chem. Mater. 15 (2003) 2181-2185.

[34] B. Chevalier, A. Wattiaux, L. Fournès, M. Pasturel, Solid State Sci. 6 (2004) 573577.

[35] B. Chevalier, M. Pasturel, J.-L. Bobet, J. Etourneau, O. Isnard, J. Sanchez Marcos, J. Rodriguez Fernandez, J. Magn. Magn. Mater. 272-276 (2004) 576-578.

[36] J. Sanchez Marcos, J. Rodriguez Fernandez, B. Chevalier, J. Magn. Magn. Mater. 310 (2007) 383-385.

[37] B. Chevalier, A. Wattiaux, J.-L. Bobet, J. Phys.: Condens. Matter 18 (2006) 17431755.

[38] B. Cornut, B. Coqblin, Phys. Rev. B 5 (1972) 4541.

[39] J. Rossat-Mignod, P. Burlet, J. Villain, H. Bartholin, W. Tcheng-Si, D. Florence, O. Vogt, Phys. Rev. B 16 (1977) 440-461.

[40] B. Lloret B. Chevalier, B. Buffat, J. Etourneau, S. Quezel, A. Lamharrar, J. Rossat-Mignod R. Calemczuk, E. Bonjour, J. Magn. Magn. Mater. 63-64 (1987) 85-87.

[41] R. Calemczuk, E. Bonjour, J. Rossat-Mignod, B. Chevalier, J. Magn. Magn. Mater. 90-91 (1990) 477-478. 


\section{Table 1}

Crystal data and structure refinement for CeIrSb and $\mathrm{CeIrSbH}_{0.8}$ with TiNiSi type, space group Pnma (No. 62), Z = 4.

\begin{tabular}{|c|c|c|}
\hline Empirical formula & CeIrSb & $\mathrm{CeIrSbH}_{0.8}$ \\
\hline Molar mass & $454.07 \mathrm{~g} / \mathrm{mol}$ & $454.1 \mathrm{~g} / \mathrm{mol}$ \\
\hline Unit cell dimensions & $a=735.07(7) \mathrm{pm}$ & $a=728.2(1) \mathrm{pm}$ \\
\hline \multirow[t]{3}{*}{ (single crystal data) } & $b=456.93(4) \mathrm{pm}$ & $b=460.4(1) \mathrm{pm}$ \\
\hline & $c=792.8(1) \mathrm{pm}_{3}$ & $c=825.9(1) \mathrm{pm}_{3}$ \\
\hline & $V=0.2663 \mathrm{~nm}^{3}$ & $V=0.2768 \mathrm{~nm}^{3}$ \\
\hline Unit cell dimensions & $a=733.5(1) \mathrm{pm}$ & $a=728.16(14) \mathrm{pm}$ \\
\hline \multirow[t]{3}{*}{ (Guinier powder data) } & $b=457.03(5) \mathrm{pm}$ & $b=460.35(6) \mathrm{pm}$ \\
\hline & $c=791.6(1) \mathrm{pm}_{3}$ & $c=825.87(2) \mathrm{pm}_{3}$ \\
\hline & $V=0.2654 \mathrm{~nm}^{3}$ & $V=0.2768 \mathrm{~nm}^{3}$ \\
\hline Calculated density & $11.37 \mathrm{~g} / \mathrm{cm}^{3}$ & $10.89 \mathrm{~g} / \mathrm{cm}^{3}$ \\
\hline Crystal size & $20 \times 40 \times 50 \mu \mathrm{m}^{3}$ & $10 \times 10 \times 70 \mu \mathrm{m}^{3}$ \\
\hline Transm. ratio (max/min) & 2.41 & 4.18 \\
\hline Absorption coefficient & $76.6 \mathrm{~mm}^{-1}$ & $73.4 \mathrm{~mm}^{-1}$ \\
\hline Detector distance & $60 \mathrm{~mm}$ & $32 \mathrm{~mm}$ \\
\hline Exposure time & $6 \min$ & $4 \min$ \\
\hline$\omega$ range; increment & $0-180^{\circ}, 1.0^{\circ}$ & - \\
\hline Integr. param. A, B, EMS & $13.5 ; 3.5 ; 0.012$ & - \\
\hline $\mathrm{F}(000)$ & 744 & 744 \\
\hline$\theta$ range & $3^{\circ}$ to $34^{\circ}$ & $5^{\circ}$ to $32^{\circ}$ \\
\hline Range in $h k l$ & $\pm 11, \pm 7, \pm 12$ & $\pm 10,-5<6,-12<8$ \\
\hline Total no. reflections & 3869 & 2588 \\
\hline Independent reflections & $601\left(\mathrm{R}_{\mathrm{int}}=0.0345\right)$ & $528\left(\mathrm{R}_{\mathrm{int}}=0.074\right)$ \\
\hline Reflections with $\mathrm{I} \geq 2 \sigma(\mathrm{I})$ & $438\left(\mathrm{R}_{\sigma}=0.0510\right)$ & $456\left(\mathrm{R}_{\sigma}=0.072\right)$ \\
\hline Data / parameters & $601 / 20$ & $528 / 20$ \\
\hline Goodness-of-fit on $\mathrm{F}^{2}$ & 0.723 & 1.15 \\
\hline \multirow[t]{2}{*}{ Final $R$ indices $[I \geq 2 \sigma(I)]$} & $\mathrm{R} 1=0.0206$ & $\mathrm{R} 1=0.0322$ \\
\hline & $w \mathrm{R} 2=0.0380$ & $w \mathrm{R} 2=0.0687$ \\
\hline \multirow[t]{2}{*}{$\mathrm{R}$ indices (all data) } & $\mathrm{R} 1=0.0355$ & $\mathrm{R} 1=0.0421$ \\
\hline & $w \mathrm{R} 2=0.0395$ & $w \mathrm{R} 2=0.0735$ \\
\hline Extinction coefficient & $0.0035(2)$ & $0.036(12)$ \\
\hline Largest diff. peak and hole & $2.82 /-3.48 \mathrm{e} / \AA^{3}$ & $1.69 /-2.45 \mathrm{e} / \AA^{3}$ \\
\hline
\end{tabular}


Table 2

Atomic positions and anisotropic displacement parameters $\left(\mathrm{pm}^{2}\right)$ of $\mathrm{CeIrSb}$ and $\mathrm{CeIrSbH}$. Pnma.

\begin{tabular}{llllllllll}
\hline Atom & Wyck. & $x$ & $y$ & $z$ & $U_{11}$ & $U_{22}$ & $U_{33}$ & $U_{13}$ & $U_{\text {eq }}$ \\
\hline CeIrSb & & & & & & & & & \\
$\mathrm{Ce}$ & $4 c$ & $0.01135(9)$ & $1 / 4$ & $0.69467(6)$ & $46(2)$ & $58(2)$ & $45(2)$ & $7(2)$ & $50(1)$ \\
$\mathrm{Ir}$ & $4 c$ & $0.29321(5)$ & $1 / 4$ & $0.41251(7)$ & $81(2)$ & $49(2)$ & $46(2)$ & $-7(2)$ & $59(1)$ \\
$\mathrm{Sb}$ & $4 c$ & $0.17924(8)$ & $1 / 4$ & $0.0871(1)$ & $64(3)$ & $34(3)$ & $39(3)$ & $1(2)$ & $45(1)$ \\
CeIrSbH & & & & & & & & \\
$\mathrm{Ce}$ & $4 c$ & $0.0134(1)$ & $1 / 4$ & $0.7012(1)$ & $62(3)$ & $83(4)$ & $100(3)$ & $6(3)$ & $82(2)$ \\
$\mathrm{Ir}$ & $4 c$ & $0.28712(7)$ & $1 / 4$ & $0.40923(6)$ & $62(3)$ & $70(3)$ & $84(3)$ & $1(2)$ & $72(1)$ \\
$\mathrm{Sb}$ & $4 c$ & $0.1710(1)$ & $1 / 4$ & $0.0834(1)$ & $68(4)$ & $63(4)$ & $79(4)$ & $7(3)$ & $70(2)$ \\
\hline
\end{tabular}

The anisotropic displacement factor exponent takes the form: $-2 \pi^{2}\left[\left(h a^{*}\right)^{2} \mathrm{U}_{11}+\cdots+2 h k a^{*} b^{*} \mathrm{U}_{12}\right] ; \mathrm{U}_{12}=$ $\mathrm{U}_{23}=0 ; \mathrm{U}_{\mathrm{eq}}$ is defined as one third of the trace of the orthogonalized $U_{\mathrm{ij}}$ tensor.

Table 3

Interatomic distances $(\mathrm{pm})$ for CeIrSb and $\mathrm{CeIrSbH}_{0.8}{ }^{\mathrm{a}}$

\begin{tabular}{lllll}
\hline & & & CeIrSb & CeIrSbH $_{0.8}$ \\
Ce: & 1 & $\mathrm{Ir}$ & 304.4 & 312.8 \\
& 2 & $\mathrm{Sb}$ & 318.7 & 320.4 \\
& 2 & $\mathrm{Ir}$ & 320.2 & 321.9 \\
& 1 & $\mathrm{Sb}$ & 330.3 & 330.4 \\
& 2 & $\mathrm{Ir}$ & 330.6 & 335.8 \\
& 2 & $\mathrm{Sb}$ & 333.1 & 339.5 \\
& 1 & $\mathrm{Sb}$ & 334.2 & 342.6 \\
& 1 & $\mathrm{Ir}$ & 349.7 & 361.4 \\
& 2 & $\mathrm{Ce}$ & 377.1 & 372.9 \\
& 2 & $\mathrm{Ce}$ & 384.0 & 404.7 \\
$\mathrm{Ir}:$ & 2 & $\mathrm{Sb}$ & 267.8 & 273.1 \\
& 1 & $\mathrm{Sb}$ & 270.8 & 279.6 \\
& 1 & $\mathrm{Sb}$ & 283.2 & 282.1 \\
& 1 & $\mathrm{Ce}$ & 304.4 & 312.8 \\
& 2 & $\mathrm{Ce}$ & 320.2 & 321.9 \\
& 2 & $\mathrm{Ce}$ & 330.6 & 330.4 \\
& 1 & $\mathrm{Ce}$ & 349.7 & 361.4 \\
$\mathrm{Sb}:$ & 2 & $\mathrm{Ir}$ & 267.8 & 273.1 \\
& 1 & $\mathrm{Ir}$ & 270.8 & 279.6 \\
& 1 & $\mathrm{Ir}$ & 283.2 & 282.1 \\
& 2 & $\mathrm{Ce}$ & 318.7 & 320.4 \\
& 1 & $\mathrm{Ce}$ & 330.3 & 335.8 \\
& 2 & $\mathrm{Ce}$ & 333.1 & 339.5 \\
& 1 & $\mathrm{Ce}$ & 334.2 & 342.6 \\
\hline
\end{tabular}

${ }^{\text {a }}$ Standard deviations are all equal or less than $0.1 \mathrm{pm}$. All distances within the first coordination spheres are listed. 


\section{Figure captions}

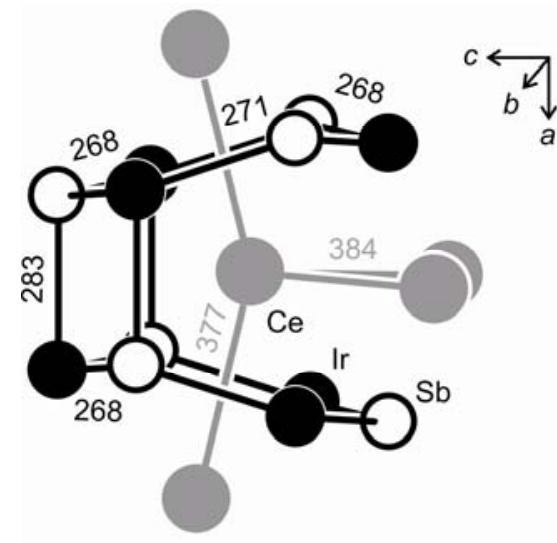

CelrSb

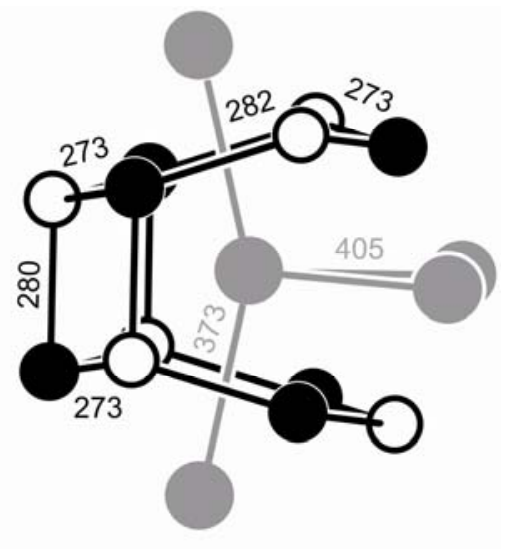

CelrSbH 0.8

Fig. 1. Near-neighbor coordination of the Ce-atoms in CeIrSb and its hydride $\mathrm{CeIrSbH}_{0.8}$. Ce, Ir, and $\mathrm{Sb}$ atoms are drawn as medium gray, black, and open circles, respectively. The puckered $\mathrm{Ir}_{3} \mathrm{Sb}_{3}$ hexagons are emphasized and relevant interatomic distances are indicated.

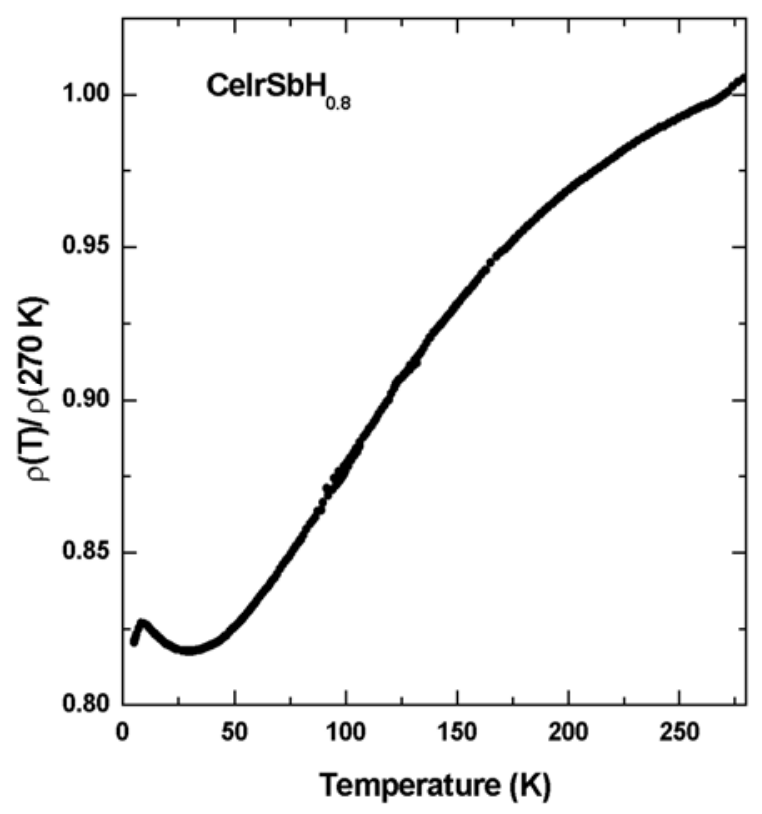

Fig. 2. Reduced electrical resistivity of $\mathrm{CeIrSbH}_{0.8}$ as a function of temperature. 


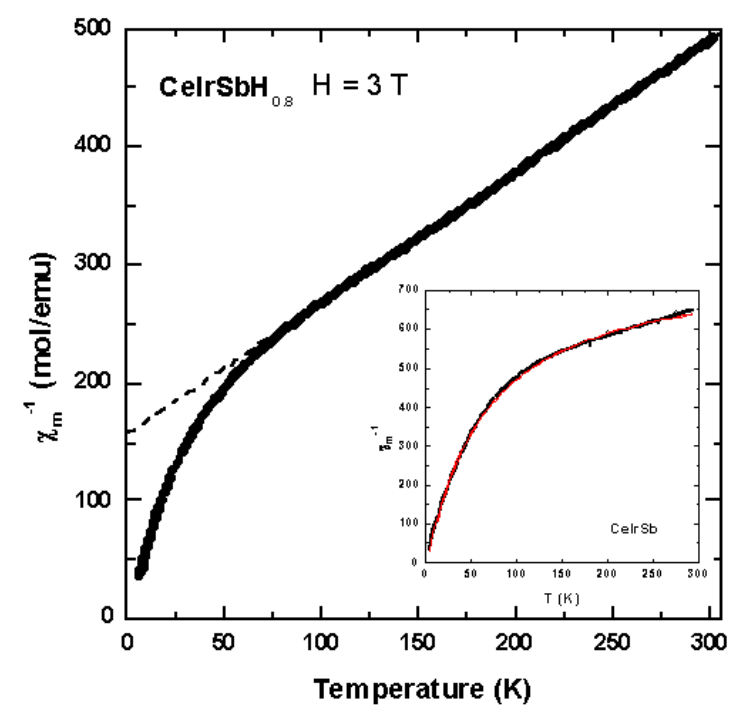

Fig. 3. Temperature dependence of the reciprocal magnetic susceptibility $\chi_{\mathrm{m}}{ }^{-1}$ of $\mathrm{CeIrSbH}_{0.8}$; the dashed line presents the Curie-Weiss law (see text). The curve $\chi_{\mathrm{m}}{ }^{-1}=$ $\mathrm{f}(\mathrm{T})$ for CeIrSb is presented in the inset; the full red line shows the modified CurieWeiss law (see text).

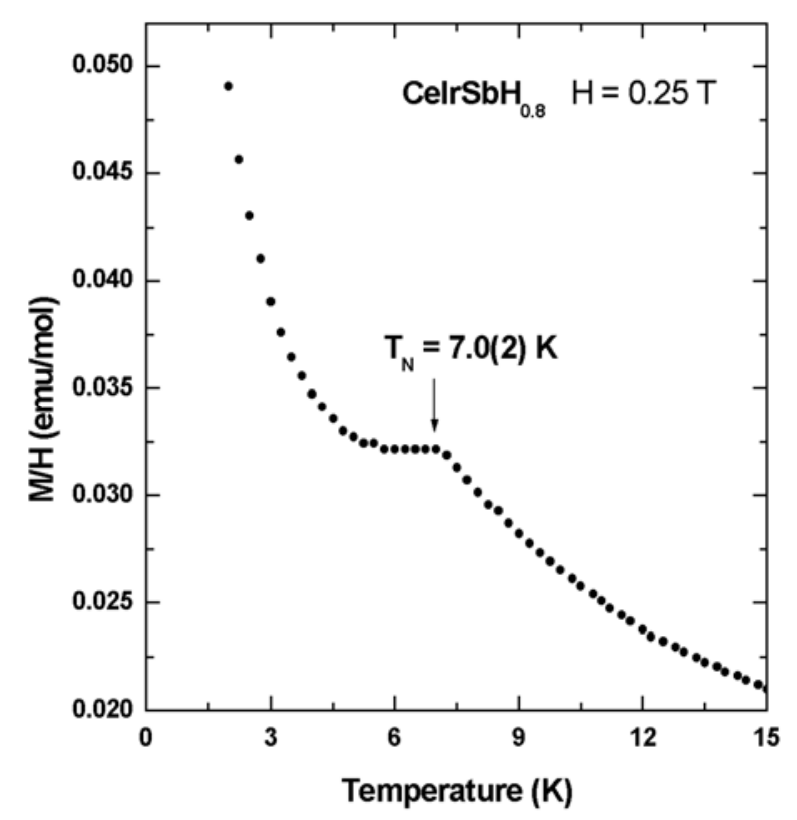

Fig. 4. Temperature dependence of the magnetization of $\mathrm{CeIrSbH}_{0.8}$ divided by the applied magnetic field $\mathrm{H}=0.25 \mathrm{~T}$. 


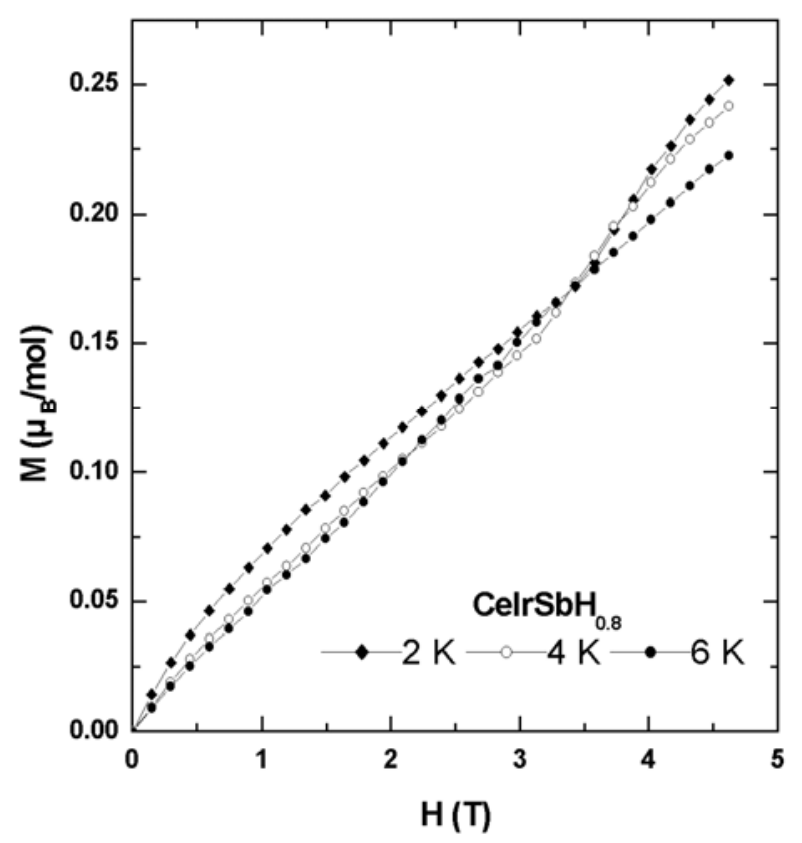

Fig. 5. Field dependence of the magnetization of $\mathrm{CeIrSbH}_{0.8}$ measured below $\mathrm{T}_{\mathrm{N}}=7.0$ $\mathrm{K}$.

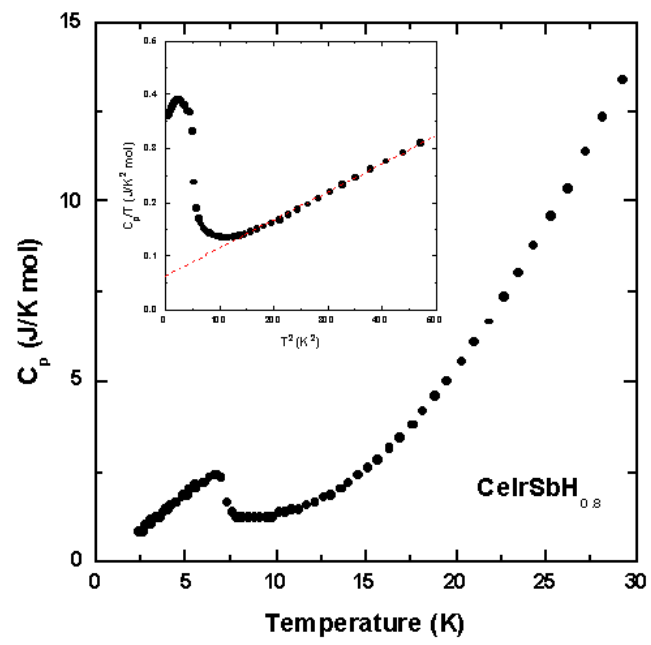

Fig. 6. Temperature dependence of the specific heat $\mathrm{C}_{\mathrm{p}}$ of the hydride $\mathrm{CeIrSbH}_{0.8}$. The curve $C_{p} / T=f\left(T^{2}\right)$ is presented in the inset; the dashed red line shows the fitting $\gamma+\beta T^{2}$ (see text). 